\title{
Analysis of the Impact of Public Hospital Corporate Governance Structure on Comprehensive Performance Based on SEM
}

\author{
Jixia Xiong, Xiaoyan Su, Li Zhang, Yanxia Lyu \\ School of Health Economics and Management, Nanjing University of Chinese Medicine
}

China

\begin{abstract}
The paper aims to deeply analyze the specific impact of public hospital corporate governance structure on the comprehensive performance in the background of the new medical reform in China, so as to provide references for promoting the reform of public hospital. The paper puts forward research hypotheses on the basis of theoretical analysis and literature review. The paper also obtains the data through the questionnaire survey in China, and acquires the impact pathway of public hospital corporate governance structure on the comprehensive performance based on the SEM (structural equation model). And the results show that the decision-making mechanism, the supervisory mechanism, the management selection and incentive mechanism and the external restraint mechanism of public hospital corporate governance structure have a significant effect on the comprehensive performance. It is suggested to promote the reform of the hospital corporate governance to improve the comprehensive performance from the above aspects.
\end{abstract}

Keywords-Public hospitals; Corporate governance structure; Comprehensive performance; Influence mechanism; Structural Equation Model (SEM)

\section{INTRODUCTION}

Public hospitals are the institutional arrangement widely adopted in the world to safeguard and improve the health of citizens. Public hospital reform has always been one of the important themes in the field of health research in the world. Clarity of property rights and scientific governance are indispensable for public hospitals to improve efficiency. It is more realistic and far-reaching to discuss how to improve hospital governance mechanism than to discuss ownership of property rights[1].Some scholars have pointed out that the current hospital governance structure in China is one of the main reasons for the inefficiency and rising costs of public hospitals[2]. The reform of public hospital governance structure is an important institutional guarantee for maintaining public welfare[3][4][5]. Since the implementation of Chinese New Medical System Reform, some positive reforms and useful exploration on corporate governance of public hospitals have been conducted and achieved initial success in China[6]. Therefore, an in-depth analysis of the impact of corporate governance structure on comprehensive performance of public hospitals is of great significance [7]. It can not only provide a basis for deepening corporate governance reform and improving the corresponding institutional arrangements in public hospitals, but also provide a reference for the

This work is supported by National Nature Scientific Foundation of China and 333 High-level Talent Cultivation Project of Jiangsu Province. government to formulate relevant health policies.

At present, most of the relevant literature studies the corporate governance structure or the comprehensive performance of hospitals from a single point of view. It can be seen in the literature that corporate governance structure has an impact on performance[8]. Our research group analysis in-depth the impact of corporate governance structure on comprehensive performance of public hospitals based on SEM. The research results can help public hospitals to improve corporate governance to enhance the comprehensive performance including economic and social benefits.

\section{RESEARCH HYPOTHESIS AND MODEL}

The core of this research is to study the impact of corporate governance structure on comprehensive performance of public hospitals. According to literature analysis and theoretical analysis, the corporate governance structure of public hospitals is divided into five elements, namely, decision-making mechanism(DES), supervision mechanism(SUV), Management Selection and Incentive Mechanism(MANG), Information disclosure mechanism(INFO) and external constraint mechanism(OUT)[9], which are all have impact on the comprehensive performance of public hospitals. The five elements are considered as 5 variables of corporate governance structure public hospitals in this paper. The comprehensive performance of public hospitals includes social benefits(SC)and economic benefits(EC), which are considered as 2 variables of hospital comprehensive performance in this paper. In the early stage, the research team has constructed a comprehensive performance index system for public hospitals including 7 indicators of social benefits and 5 indicators economic benefits[10]. Based on the index system, social benefits(SC) and economic benefits(EC) SC of public hospitals can be expressed by 7 indicators including the number of medical services, the quality of medical services, the efficiency of medical services, scientific research capacity, social satisfaction, and the EC of public hospitals can be shown by 5 indicators including solvency, operational capacity, efficiency capacity, cost control capacity and development capacity. We take these indicators as observation variables.

On the basis of previous research results, this study identified DES, SUV, MANG, INFO, OUT as independent variables, SC and EC as dependent variables. Management is the key to corporate governance in public hospitals, which is related to the operation and development of the whole hospital. Literature studies show that there is a significant relationship 
between organizational performance and management effort. Management effort determines the social and economic benefits of public hospitals[11]. Whether the corporate governance structure is perfect or not is the key to determine the management effort. In this study, management effort was set as mediating variables, namely FIGHT, which played a mediating role in the impact of corporate governance structure on comprehensive performance in public hospitals. Based on the previous literature review and theoretical analysis, research hypothesis is presented in Table I.

\section{TABLE I. RESEARCH HYPOTHESES}

\begin{tabular}{cl}
\hline hypotheses & Hypothetical content \\
\hline H1a & DES has a significant impact on SC \\
H1b & DES has a significant impact on EC \\
H2a & SUV has a significant impact on SC \\
H2b & SUV has a significant impact on EC \\
H3a & MANG has a significant impact on SC \\
H3b & MANG has a significant impact on EC \\
H4a & INFO has a significant impact on SC \\
H4b & INFO has a significant impact on EC \\
H5a & OUT has a significant impact on SC \\
H5b & OUT has a significant impact on EC \\
H6a & DES has a significant impact on FIGHT \\
H6v & SUV has a significant impact on FIGHT \\
H6c & MANG have a significant impact on FIGHT \\
H6d & INFO has a significant impact on FIGHT \\
H6e & OUT has a significant impact on FIGHT \\
H6f & FIGHT have a significant impact on SC \\
H6g & FIGHT have a significant impact on EC \\
\hline
\end{tabular}

Based on the research hypothesis, we construct the research model of the impact of corporate governance structure on comprehensive performance in public hospitals, and then validate the research hypothesis by SEM.

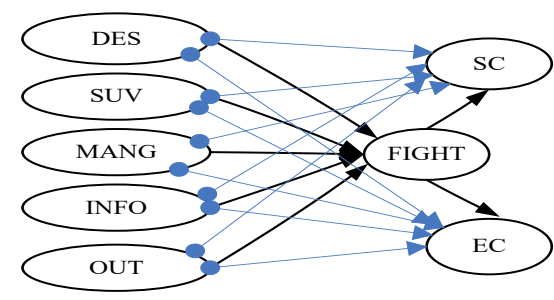

Fig. 1. Research model

\section{QUESTIONNAIRE SURVEY AND ANALYSIS}

This study used a questionnaire survey to get date and conduct empirical research, in order to provide a basis for SEM and validate the research hypothesis.

\section{A. Questionnaire issuance}

In this study, the research areas selected all are typical areas of China, in which the public hospitals have implemented corporate governance structure reform or governance structure is clear in recent years. Five typical cities including Nanjing, Zhenjiang, Suzhou, Wuxi and Shanghai has been selected as the main research areas, and their health administrative departments also have been investigated,3-4 large public hospitals have been selected as sample hospitals in each city. In addition, Xuzhou, Changzhou, Nantong, Hangzhou, Fuzhou and other places have been selected as the areas of this survey, increasing the geographical coverage and effectiveness of the questionnaire. The specific survey respondents are as follows: middle and senior management personnel of public hospitals; doctors of public hospitals; the administrative staffs of the Health Administrative Department; health management policy research experts. A total of 600 questionnaires were handed out in this survey and 572 questionnaires were returned, including 520 valid questionnaires and 52 invalid questionnaires. The effective questionnaire recovery rate was $90.91 \%$. Based on the collected questionnaires, SPSS 19.0 was used to analyze the reliability and validity of all questionnaires.

\section{B. Reliability analysis of the questionnaires}

The reliability and stability of the questionnaire results can be measured by the reliability test. This study used Cronbach's $\alpha$ coefficients to test the reliability of the scale. According to the summarization of most of the literature, if the Cronbach's $\alpha$ coefficient is greater than 0.8 , the reliability is considered acceptable. According to the sample data, the Cronbach's $\alpha$ coefficients of public hospitals' comprehensive performance, public hospital corporate governance structure and management effort level are measured by SPSS19.0, and the Cronbach's $\alpha$ coefficients are $0.820,0.885$, and 0.846 , respectively. They are within the acceptance range and the reliability of the scale is acceptable; then the Cronbach's $\alpha$ coefficient of the total number of 32 items is measured, and the Cronbach's $\alpha$ coefficient of the overall scale is 0.915 , which indicates that the overall reliability of the sample is good and the survey has a good internal consistency.

\section{Validity analysis of the questionnaire}

The questionnaire of this study is designed based on the reference of relevant literature and theory. The study did pre-study before the start of formal research. The items in the questionnaire have been revised several times based on the results of the pre-study and feedback and combined with the guidance of health management experts. So the content validity and the criterion validity is ensured.

Structural validity is mainly used to verify that the scale truly measures the variables. Factor analysis is often used for the examination of structural validity. This study uses $K M O$ sample measures and Bartlett spherical Cronbach's $\alpha$ tests to testify whether the scale data is suitable for factor analysis.

(1) The $K M O$ value of the overall scale is 0.900 , and the significant probability of the chi-square statistical value of the Cronbach's $\alpha$ spherical test is $0.000(<0.05)$, indicating that the correlation between the items is good and suitable for factor analysis.

(2) The $K M O$ value of the comprehensive performance scale is 0.894 , and the significant probability of the chi-square statistical value of the Cronbach's $\alpha$ spherical test is 0.000 $(<0.05)$, indicating that the correlation between the items is good and suitable for factor analysis. The study does further analysis of the 12 items of the comprehensive performance scale, and extract 2 common factors according to the eigenvalue greater than 1. Seven original SC indicators are extracted as a factor F1. Five original EC indicators are 
extracted as a factor F2. The factor load values of these 12 factors are all exceed 0.5 , so all the indicators are retained.

(3) The $K M O$ value of the corporate governance scale of the public hospital is 0.857 , and the significant probability of the chi-square statistical value of the Cronbach's $\alpha$ spherical test is $0.000(<0.05)$, indicating that the correlation between the items is good and suitable for factor analysis. The study does the factor analysis of 16 items of the corporate governance scale, and extract 4 common factors according to the eigenvalue greater than 1 . SUV and INFO are extracted as a common factor F2, and the other three factors F1, F3, and F4 are DES, MANG and OUT, which are consistent with the original classification. Each item has a higher load value (all greater than 0.6), indicating that the attribute of each item is stronger, and the difference between the factors is better, so all indicators are retained. Through the results of factor analysis, the corporate governance indicators of public hospitals are adjusted to four, DES, SUV ,MANG and OUT.

(4)The KMO value of the management effort scale is 0.794 , and the significant probability of the chi-square statistical value of the Cronbach's $\alpha$ spherical test is $0.000(<0.05)$, indicating that the correlation between the items is good and suitable for factor analysis. Further factor analysis is carried out on the 4 items of the management effort scale, and 1 common factor is extracted according to the eigenvalue greater than 1 . The load value of each item is greater than 0.8 , indicating that the difference between indicators is effective.

\section{Indicator system and hypothesis correction}

According to the results of the factor analysis, the original indicators are revised and summarized as follows:

(1)Independent variable: corporate governance of public hospitals is adjusted from the original five indicators to four indicators, DES, SUV ,MANG and OUT.

(2)Dependent variable comprehensive performance indicators and intermediary variables management effort indicators are unchanged.

According to the correction of the above indicators, the original model hypotheses are amended, see Table II.

TABLE II. SUMMARY OF REVISED RESEARCH HYPOTHESIS

\begin{tabular}{cl}
\hline hypothesis & Content of hypothesis \\
\hline H1a & DES has a significant impact on SC \\
H1b & DES has a significant impact on EC \\
H2a & SUV has a significant impact on SC \\
H2b & SUV has a significant impact on EC \\
H3a & MANG has a significant impact on SC \\
H3b & MANG has a significant impact on EC \\
H4a & OUT has a significant impact on SC \\
H4b & OUT has a significant impact on EC \\
H5a & DES has a significant impact on FIGHT \\
H5b & SUV has a significant impact on FIGHT \\
H5c & MANG has a significant impact on FIGHT \\
H5d & OUT has a significant impact on FIGHT \\
H5e & FIGHT has a significant impact on the SC \\
H5f & FIGHT has a significant impact on the EC \\
\hline
\end{tabular}

\section{SEM ANALYSIS AND TEST RESULTS}

The purpose of this study is to explore the influence of corporate governance structure of public hospitals on comprehensive performance. There are many independent variables and dependent variables, observation variables and latent variables in this study, so SEM is adopted for analysis.

SEM is divided into two categories: one is the measurement model, which mainly reflects the relationship between observable variables and latent variables through confirmatory factor analysis. The second is the structural model, which is a method of path analysis to discuss the relationship between variables. In this study, we verify the hypothesis by the measurement model, and then verify the correlation between the variables through the structural model.

\section{A. Constructing SEM}

(1) Model building. In the previous section, we have defined the causal relationship between variables, which will not be repeated here.

(2) Model recognition. Structural equation analysis can identify a particular model by calculating the degree of freedom of the model, $d . f=\frac{(p+q)(p+q+1)}{2}-t$, d.f is the degree of freedom of the model, $\mathrm{p}$ is the number of exogenous observation variables, $\mathrm{q}$ is the number of endogenous observation variables, and $\mathrm{t}$ is the sum of the estimated parameters of the model. When d.f $>1$, the model can be fully recognized. In this model, $\mathrm{p}+\mathrm{q}=32, \mathrm{t}=92$, d. $\mathrm{f}=448$, so it can be fully recognized.

TABLE III. CFA model factor loading of the overall scale

\begin{tabular}{|c|c|c|c|c|}
\hline Variable & Index & Estimated Value & $\begin{array}{c}\text { Standardized } \\
\text { Estimates }\end{array}$ & T-value \\
\hline \multirow[t]{4}{*}{ DES } & DES1 & 1.00 & 0.59 & 14.20 \\
\hline & DES2 & 0.70 & 0.57 & 13.62 \\
\hline & DES3 & 0.83 & 0.92 & 26.04 \\
\hline & DES4 & 0.91 & 0.92 & 26.11 \\
\hline \multirow[t]{6}{*}{ SUV } & SUV1 & 0.76 & 0.60 & 14.17 \\
\hline & SUV2 & 0.96 & 0.79 & 20.03 \\
\hline & SUV3 & 1.14 & 0.79 & 20.24 \\
\hline & SUV4 & 0.99 & 0.69 & 16.74 \\
\hline & SUV5 & 1.02 & 0.65 & 15.42 \\
\hline & MANG1 & 0.90 & 0.77 & 20.09 \\
\hline \multirow[t]{3}{*}{ MANG } & MANG2 & 0.69 & 0.80 & 20.89 \\
\hline & MANG3 & 0.73 & 0.82 & 21.89 \\
\hline & MANG4 & 0.83 & 0.79 & 20.69 \\
\hline \multirow[t]{3}{*}{ OUT } & OUT1 & 0.86 & 0.71 & 17.35 \\
\hline & OUT2 & 1.10 & 0.84 & 21.84 \\
\hline & OUT3 & 1.06 & 0.80 & 20.67 \\
\hline \multirow[t]{4}{*}{ FIGHT } & FIGHT1 & 0.79 & 0.81 & 21.53 \\
\hline & FIGHT2 & 1.11 & 0.85 & 23.09 \\
\hline & FIGHT3 & 0.61 & 0.77 & 20.15 \\
\hline & FIGHT4 & 0.66 & 0.81 & 21.62 \\
\hline \multirow[t]{7}{*}{$\mathrm{SC}$} & $\mathrm{SC} 1$ & 0.72 & 0.65 & 15.98 \\
\hline & $\mathrm{SC} 2$ & 1.12 & 0.81 & 21.52 \\
\hline & SC3 & 1.06 & 0.78 & 20.28 \\
\hline & SC4 & 0.89 & 0.60 & 14.29 \\
\hline & SC5 & 0.67 & 0.58 & 13.86 \\
\hline & SC6 & 1.38 & 0.76 & 19.55 \\
\hline & SC7 & 0.97 & 0.64 & 15.74 \\
\hline \multirow[t]{5}{*}{$\mathrm{EC}$} & EC1 & 0.71 & 0.67 & 16.68 \\
\hline & $\mathrm{EC} 2$ & 0.73 & 0.77 & 20.22 \\
\hline & EC3 & 1.09 & 0.80 & 21.23 \\
\hline & EC4 & 0.94 & 0.76 & 19.55 \\
\hline & EC5 & 1.31 & 0.80 & 21.10 \\
\hline
\end{tabular}


(3) Model evaluation. On the basis of exploratory factor analysis, we verify the relationship between latent variables and observed variables by the confirmatory factor analysis to prove the rationality of the proposed hypothesis. By using Lisrel 9.20 software, CFA model factor loading of overall scale is obtained as shown in Table III.

The loading value of each factor is higher than 0.5 , which is within the acceptable range. That is, each factor can explain the corresponding latent variable to a certain extent, so all indexes are retained.

The fitting results of the measurement item CFA of the analysis model of the influence of the corporate governance structure on comprehensive performance of public hospitals are shown in table IV.

According to the path established by the hypothesis, the fitting conditions of various indicators are shown in table V.
TABLE iV. Estimation of Specific Path Coefficient Parameters

\begin{tabular}{|c|c|c|c|c|}
\hline Variable $\leftarrow$ Factor & $\begin{array}{l}\text { Estimated } \\
\text { Value }\end{array}$ & $\begin{array}{l}\text { Standardized } \\
\text { estimation value }\end{array}$ & \multicolumn{2}{|c|}{ T-value } \\
\hline FIGHT $\leftarrow$ DES & 0.10 & 0.09 & 2.37 & $>1.96$ \\
\hline FIGHT $\leftarrow$ SUV & 0.14 & 0.14 & 2.66 & $>1.96$ \\
\hline FIGHT $\leftarrow \mathrm{MANG}$ & 0.20 & 0.20 & 3.09 & $>1.96$ \\
\hline FIGHT $\leftarrow$ OUT & 0.42 & 0.42 & 6.34 & $>1.96$ \\
\hline $\mathrm{SC} \leftarrow$ FIGHT & 0.27 & 0.27 & 4.95 & $>1.96$ \\
\hline $\mathrm{EC} \leftarrow$ FIGHT & 0.19 & 0.20 & 3.54 & $>1.96$ \\
\hline $\mathrm{SC} \leftarrow \mathrm{DES}$ & 0.06 & 0.06 & 1.52 & $<1.96$ \\
\hline $\mathrm{EC} \leftarrow \mathrm{DES}$ & 0.00 & 0.00 & 0.08 & $<1.96$ \\
\hline $\mathrm{SC} \leftarrow \mathrm{SUV}$ & 0.16 & 0.16 & 3.37 & $>1.96$ \\
\hline $\mathrm{EC} \leftarrow \mathrm{SUV}$ & 0.05 & 0.05 & 1.00 & $<1.96$ \\
\hline $\mathrm{SC} \leftarrow \mathrm{MANG}$ & 0.26 & 0.26 & 4.20 & $>1.96$ \\
\hline $\mathrm{EC} \leftarrow \mathrm{MANG}$ & 0.39 & 0.39 & 6.00 & $>1.96$ \\
\hline $\mathrm{SC} \leftarrow \mathrm{OUT}$ & 0.20 & 0.20 & 3.12 & $>1.96$ \\
\hline $\mathrm{EC} \leftarrow \mathrm{OUT}$ & 0.20 & 0.20 & 3.03 & $>1.96$ \\
\hline
\end{tabular}

TABLE V. THE FITTING RESULTS OF THE MEASUREMENT ITEM CFA OF THE ANALYSIS MODEL

\begin{tabular}{llllllllll}
\hline Fitting index & $\chi 2 / \mathrm{df}$ & GFI & AGFI & NFI & NNFI & CFI & RMR & RMSEA & P \\
\hline & 4.5 & 0.80 & 0.77 & 0.93 & 0.94 & 0.95 & 0.052 & 0.082 & $>0.05$ \\
\hline
\end{tabular}

From Table V, it can be seen that the T-value of 3 path coefficient is less than 1.96, indicating that the correlation between the two variables is not significant. So we take model correction on the initial model(model M1).

\section{B. Model correction}

(1) Model correction one. Taking T-value $(>1.96)$ as the standard, we delete the insignificant paths: SC $\leftarrow$ DES , $\mathrm{EC} \leftarrow \mathrm{DES}, \mathrm{EC} \leftarrow \mathrm{SUV}$, and then fit the model again and get the model M2.
(2) Model correction two. According to Modifications Indices (MI.), we increase the $\mathrm{EC} \rightarrow \mathrm{SC}$ path, so that the chi-square value of 119.73 can reduced, and then we can get the model M3.

(3) Model correction three. Taking T-value $(>1.96)$ as the standard, we delete the insignificant paths: $\mathrm{SC} \leftarrow \mathrm{MANG}$, $\mathrm{SC} \leftarrow \mathrm{OUT}$, and then fit the model again and get the model M4.

The fitting conditions of model M1, M2, M3 and M4 are summarized in table VI:

TABLE VI. COMPARISON OF FITTING EXPONENT OF THE MODEL BEFORE AND AFTER MODIFICATION

\begin{tabular}{llllll}
\hline Fitting index & Model M1 & Model M2 & Model M3 & Model M4 & Model acceptance criteria \\
\hline$\chi^{2}$ & 2132.79 & 2133.48 & 2022.00 & 2021.81 & - \\
df & 444 & 447 & 446 & 448 & -- \\
NC Value: $\chi^{2 / \text { df }}$ & 4.80 & 4.77 & 4.53 & 4.51 & 5 \\
GFI & 0.80 & 0.80 & 0.80 & 0.80 & $>0.9$ \\
AGIF & 0.79 & 0.76 & 0.77 & 0.77 & $>0.8$ \\
RMSEA & 0.09 & 0.09 & 0.08 & 0.08 & $<0.08$ \\
RMR & 0.06 & 0.07 & 0.05 & 0.05 & $<0.08$ \\
NFI & 0.93 & 0.93 & 0.93 & 0.93 & $>0.90$ \\
NNFI & 0.93 & 0.94 & 0.94 & 0.94 & $>0.90$ \\
IFI & 0.94 & 0.94 & 0.95 & 0.95 & $>0.90$ \\
CFI & 0.94 & 0.94 & 0.95 & 0.95 & $>0.90$ \\
PNFI & 0.83 & 0.84 & 0.84 & 0.84 & $>0.50$ \\
\hline
\end{tabular}

After correction, it is found that

so the overall fitting effect of the model M4 is acceptable. We $\chi 2$ ddf,AGIF,RMSEA,RMR,NNFI,IFI,CFI,PNFI, $\quad$ AGIF, RMSEA, RMR, NNFI, IFI, CFI and PNFI had changed. According to the standard, the smaller $\chi 2$ and RMR values, the better the effect; the larger the AGIF and PNFI values, the better the effect. Therefore, the model M4 is superior to M3, M2 and M1. In the model M4, most fitting exponents are within the standard range, only GFI and AGIF are slightly different from the standard, but they are similar and acceptable,

\section{SEM test results}

According to the calculation of the path coefficient of the final model, we can calculate the total degree of influence between the variables. The direct effect can be directly obtained from the path coefficient in the structural equation, the indirect effect can be obtained by calculation, and the 
summation effect is the sum of the indirect effect and the direct effect (Table VII). The final fitting diagram of the SEM is shown in Fig. 2.

TABLE VII. SUMMATION EFFECT

\begin{tabular}{lllllll}
\hline & \multicolumn{1}{c}{ DES } & SUV & MANG & OUT & FIGHT & EC \\
\hline FIGHT & 0.10 & 0.13 & 0.20 & 0.42 & 0.00 & 0.00 \\
SC & 0.03197 & 0.211561 & 0.31594 & 0.266574 & 0.20 & 0.63 \\
EC & 0.019 & 0.4247 & 0.038 & 0.2898 & 0.19 & 0.00 \\
\hline
\end{tabular}

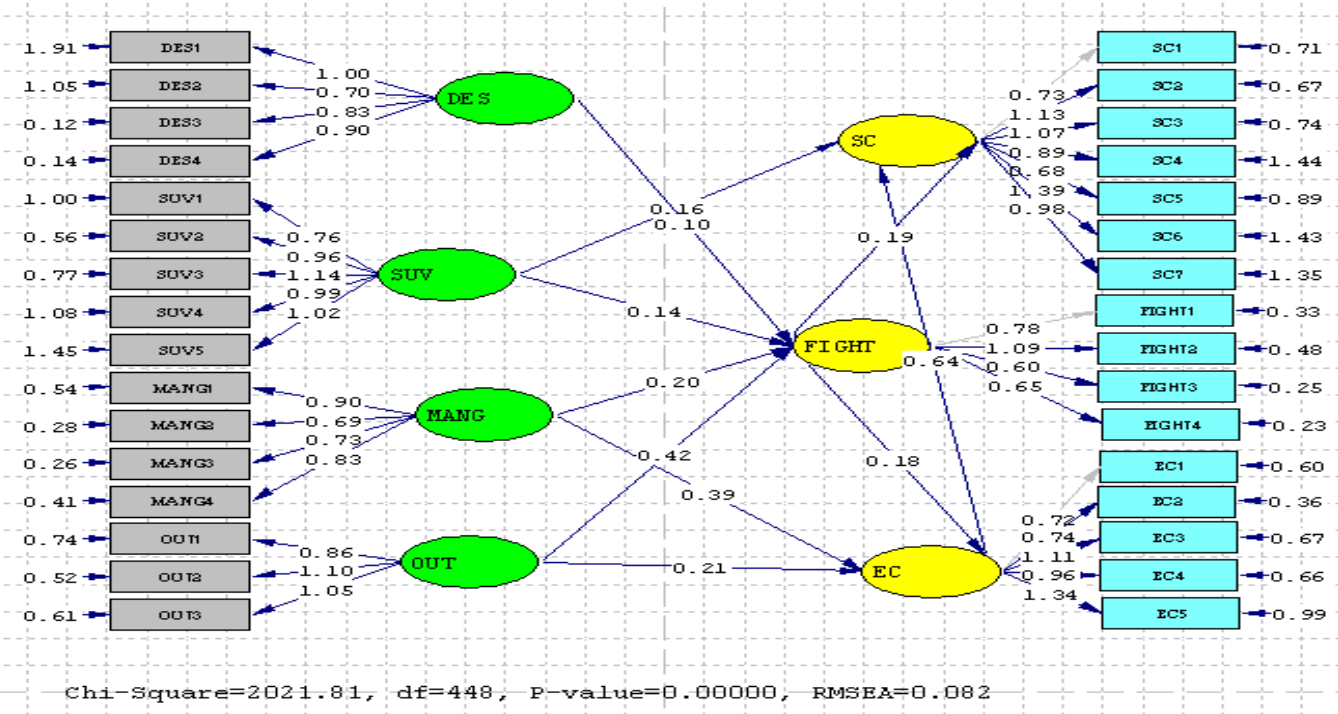

Fig. 2 The final fitting diagram of the SEM

The direct path coefficient T-value $(>1.96)$ is used as the criterion to verify the hypothesis. The results are shown in Table VIII:

TABLE VIII. Research hypothesis summary

\begin{tabular}{lll}
\hline Hypothesis & Hypothetical content & Result \\
\hline $\mathrm{H} 1 \mathrm{a}$ & DES has a significant impact on SC & Nonsupport \\
$\mathrm{H} 1 \mathrm{~b}$ & DES has a significant impact on EC & Nonsupport \\
$\mathrm{H} 2 \mathrm{a}$ & SUV has a significant impact on SC & Support \\
$\mathrm{H} 2 \mathrm{~b}$ & SUV has a significant impact on EC & Nonsupport \\
$\mathrm{H} 3 \mathrm{a}$ & MANG has a significant impact on SC & Nonsupport \\
$\mathrm{H} 3 \mathrm{~b}$ & MANG has a significant impact on EC & Support \\
$\mathrm{H} 4 \mathrm{a}$ & OUT has a significant impact on SC & Nonsupport \\
$\mathrm{H} 4 \mathrm{~b}$ & OUT has a significant impact on EC & Support \\
$\mathrm{H} 5 \mathrm{a}$ & DES has a significant impact on FIGHT & Support \\
$\mathrm{H} 5 \mathrm{~b}$ & SUV has a significant impact on FIGHT & Support \\
$\mathrm{H} 5 \mathrm{c}$ & MANG has a significant impact on FIGHT & Support \\
$\mathrm{H} 5 \mathrm{~d}$ & OUT has a significant impact on FIGHT & Support \\
$\mathrm{H} 5 \mathrm{e}$ & FIGHT has a significant impact on SC & Support \\
$\mathrm{H} 5 \mathrm{f}$ & FIGHT has a significant impact on EC & Support \\
\hline
\end{tabular}

Except H1a, H1b, H2b, H3a and H4a have not been verified, all the other hypotheses have passed the verification.

\section{Result analysis}

(1) H1a the T-value of path coefficient between DES and $\mathrm{SC}$ is $1.52, \mathrm{H} 1 \mathrm{~b}$ the T-value of path coefficient between DES and EC is 0.08 ,both are less than 1.96 , so neither hypothesis is significant. However, the T-value of path coefficient between DES and FIGHT is 1.52, DES exerts indirect influence on comprehensive performance of public hospitals through FIGHT, and FIGHT play a completely mediating role.

(2) H2b the T-value of path coefficient between SUV and $\mathrm{EC}$ is $1(<1.96)$, so it is not significant. However, the T-value of path coefficient between SUV and FIGHT is 0.14 , SUV exert indirect influence on EC of public hospitals through FIGHT, and FIGHT play a completely mediating role.

(3) $\mathrm{H} 3 \mathrm{a}$ the T-value of path coefficient between MANG and $\mathrm{SC}$ is $0.02(<1.96)$, it is not significant. However, the T-value of path coefficient between MANG and FIGHT is 0.2 , MANG exert indirect influence on SC of public hospitals through FIGHT, and FIGHT play a completely mediating role.

(4) H4a the T-value of path coefficient between OUT and $\mathrm{SC}$ is $1(<1.96)$, so it is not significant. However, the T-value of path coefficient between OUT and FIGHT is 0.42 , OUT exert indirect influence on SC of public hospitals through FIGHT, and FIGHT play a completely mediating role.

According to the table of summation effect and the size of the path coefficient, it can be judged that the degree of influence on the social and economic benefits in the comprehensive performance is from large to small as follows:

(1) The order of influence on social benefits is as follows: MANG, OUT, SUV, FIGHT and DES.

(2) The order of influence on economic benefits is as follows: SUV, OUT,FIGHT, MANG, and DES.

These path coefficients and rankings can reflect the influence of various factors on the comprehensive performance, 
and provide a reference basis for the subsequent research to put forward effective suggestions.

\section{CONCLUSIONS AND SUGGESTIONS}

\section{A. Conclusions}

This study proposes the hypothesis of the impact of corporate governance structure on comprehensive performance of public hospitals. The study obtains relevant data through questionnaire survey, and processes the data by reliability and validity analysis, as well as SEM, so as to verify the hypothesis. The following conclusions are drawn from the study:

(1)The corporate governance structure of public hospitals determines the decision-making mechanism, the supervisory mechanism, the management selection and incentive mechanism and the external restraint mechanism. These factors can affect the management's efforts and behavior, thus affect the comprehensive performance of public hospitals including social benefits and economic benefits.

(2)The decision-making mechanism, the supervisory mechanism, the management selection and incentive mechanism and the external restraint mechanism all have significant impact on the comprehensive performance of public hospitals. Among them, the management selection and incentive mechanism and external restraint mechanism have the most significant impact on social benefits, and supervision mechanism and external restraint mechanism have the most significant impact on economic benefits. Therefore, we can optimize the above corporate governance system arrangements to promote the improvement of comprehensive performance of public hospitals

\section{B. Suggestions}

According to the research results, we suggest that public hospitals improve the following corporate governance system arrangement in order to improve the comprehensive performance. First, improve the decision-making mechanism of public hospitals, ensure that stakeholders participate in the decision-making of hospitals, and make sure that hospitals give consideration to both economic benefits and social benefits. Second, improve the supervisory mechanism of public hospitals, improve the rules and regulations and audit system, and avoid the performance of profit to ensure the sustained and effective economic benefits and public welfare; Third, set up a system of open recruitment, reasonable assessment and salary to improve the management recruitment and incentive mechanism; Fourth, deepen separation of management and operation, strengthen market competition, and improve external restraint mechanisms.

\section{ACKNOWLEDGEMENT}

Thanks for the support from National Nature Scientific Foundation of China and "333 High-level Talent Cultivation Project” of Jiangsu Province.

\section{REFERENCES}

[1] Zhang Lulu, Li Jin, Xu Zuming. Advanced Hospital Management Science[M]. Shanghai: The Second Military Medical University press, 2003.

[2] Li Weiping, Zhou Haisha, Liu Neng. General Report of Governance Structure of Public Hospitals[J]. Chinese Hospital Management,2005,25(8):5-8.

[3] Zheng Daxi. An Economic Analysis of the Public Welfare Nature and Running Performance Evaluation of Public Hospitals[J].Medicine and Society,2010,23(11): 51-53.

[4] Xiong Jixia, Xu Aijun. Suggestion on Reform of State-Owned Hospitals Governance Structure Based on its Commonweal Character [J]. Chinese Health Service Management, 2010,27(11):747-748,767.

[5] Dong Yunping, Wang Yuqin, Lu Hui, Luo Wujin. Analysis of Path Dependence of Public Welfare Regression of Public Hospitals under the New Medical Reform [J].Chinese Hospital Management,2010,30(7):3-5.

[6] Zhang Li. The research on the effect of corporate governance to public welfare in public hospital[D].Nanjing University of Chinese Medicine ,2013.

[7] Xiong Jixia, Su Xiaoyan. Research the Context of the New Health Care Reform about Influence of the Corporate Governance Structure on Comprehensive performance of Public Hospitals[J].Chinese Health Service Management,2013, 30(11): 848-852.

[8] Li Shiguo, Shi Hongwei. The key factor for improving public hospital performance is to promote governance structure-the revelation from Hong Kong public hospital governance structure[J]. Chinese Hospital,2010,14(17):23-26.

[9] Xiong Jixia, Zhang Li, Gao Lina. Impact analysis of public hospital corporate governance on their public welfare[J].Chinese Journal of Hospital Administration, 2016, 32(4):314-318.

[10] Xiong Jixia, Zhang Zhiqiang. Construction of Comprehensive Performance Evaluation Index System of Public Hospitals Based on Public Welfare[J] .Social Sciences in Guangxi, 2014,(6):165-169.

[11] Xiong Jixia, Su Xiaoyan. Designing the reform on corporate governance to improve the comprehensive performance of public hospitals based on principal-agent theory[J]. Chinese Health Service Management, 2016,33(2):87-90. 